PII: S0045-6535(97)00284-1

\title{
DETERMINATION OF ARYLAMINE COMPOUNDS IN WASTE WATER USING SOLID-PHASE EXTRACTION AND REVERSED-PHASE HIGH PERFORMANCE LIQUID CHROMATOGRAPHY
}

\author{
Shu-Li Zhao ${ }^{1}$, Fu-Sheng Wei ${ }^{1}{ }^{*}$, Han-Fa Zou ${ }^{2}$ and Xiao-Bai $\mathrm{Xu}^{3}$ \\ ${ }^{1}$ China National Environmental Monitoring Center, Beisihuan Dong Lu, Beijing, 100029, China \\ ${ }^{2}$ Dalian Institute of Chemical Physics, Chinese Academy of Sciences, Dalian, 116012, China \\ ${ }^{3}$ Research Center for Eco-Environmental Sciences, Chinese Academy of Sciences, Beijing, 100085, China
}

(Received in Germany 16 June 1997; accepted 15 July 1997)

\section{ABSTRACT}

A simple, rapid and sensitive method for determining 10 arylamine compounds in wastewater sample using solid-phase extraction (SPE) and high performance liquid chromatography (HPLC) with UV detection is developed. An SPE procedure is described for extracting and preconcentrating the arylamines on Sep-Pak C18 cartridges with methanol as solvent. A mobile phase gradient of acetonitrile-water was used for the separation and determination of the arylamines. The detection limits of the compounds were in the range of $0.1-1.5 \mu \mathrm{g} / \mathrm{L}$. Recoveries were $84-107 \%$ for 10 arylamines at concentration levels of $20 \mu \mathrm{g} / \mathrm{L}$ with relative standard deviations of less than $8 \%(n=4)$. (C) 1997 Published by Elsevier Science Ltd

\section{INTRODUCTION}

Arylamine compounds are valuable chemicals in many areas of industry and research, in the meantime, they are also an important class of environmental water pollutants. These compounds may enter the environment directly as industrial discharges or indirectly from pesticide degradation. Many of these compounds are highly toxic and carcinogenic [1]. Some of the arylamines have been included in the present priority pollutant list of National Environmental Protection Agency in China. For this reason, there is a need for relatively specific, sensitive procedures which can be utilized in the routine analysis for these compounds in the environment, especially in aqueous environmental samples.

Several arylamine compounds such as aniline, benzidine and halogenated anilines in environmental waters were determined by liquid chromatography [2-7]. However, few liquid chromatographic procedures have been established to determine a large group of arylamine compounds in polluted waters. With the rapid 
growth of industry, it is an urgent task to develop a convenient and sensitive method for monitoring toxic and carcinogenic arylamines in industrial wastewater

Sample preparation is an important step in the analysis of complex water matrices for such organic pollutants as arylamines. Considerable attention is now being paid to SPE as a way to isolate and concentrate desired components from a sample matrix. As an inexpensive and convenient method for sample extraction and preconcentration, SPE is suitable for extracting arylamines from environmental water samples [7-9] The aim of the present work was to develop a method based on SPE and HPLC, that allowed the separation and detection of arylamines in wastewater generated from the manufacture of dyes.

\section{EXPERIMENTAL}

\section{Apparatus and Materials}

All HPLC studies were performed using a SP-8800 liquid chromatograph (Spectro-Physics, USA) equipped with a SP-8800 ternary gradient pump, a Hypersil BDS, $5 \mu \mathrm{m}$ particle diameter, $200 \times 4.0 \mathrm{~mm}$ stainless steel column, a GL605 injector valve (10 $\mathrm{L}$ loop), a spectra $100 \mathrm{UV}-\mathrm{Vis}$ detector. A WDL-95 workstation (Dalian, China) was utilized to control the system and for acquisition and analysis of data. Disposable ODS reversed phase cartridges (500 mg, Sep-Pak C18, J \& W, USA) and an SPE vacuum manifold system (Dalian Chromatography Research Center, Dalian, China) were used to extract and preconcentrate the arylamines from water samples

\section{Chemicals}

All reagents were "Analytical reagents" grade. Methanol, acetonitrile, aniline, benzidine and quinoline were obtained from Beijing Chemical Plant (Beijing. China) Other arylamine compounds studied were obtained from Sigma (St. Louis, MO, USA)

Deionized water was purified in a Milli-Q filtration system (Millipore, USA) to obtain LC-grade water for preparing mobile phases and standard solutions. HPLC mobile phases were filtered through a $0.45-\mu \mathrm{m}$ HA filter (Millipore) and degassed.

Stock standard solutions of the arylamines were prepared by dissolving arylamines in methanol, the concentrations of the analytes being $100 \mathrm{mg} / \mathrm{L}$. A working composite standard solution was prepared by combining an aliquot of each of the stock solutions and diluting the mixture with methanol. These solutions were stored in dark glass bottles at $4^{\circ} \mathrm{C}$

\section{Sample Collection}

Wastewater samples were collected from the aqueous effluents (after secondary treatment) in a dye processing plant in Dalian city. All samples were collected during winter 1996 and were stored in a 
refrigerator at $4^{\circ} \mathrm{C}$ until extraction. All samples were extracted within 24 hours of collection and then analyzed.

\section{SPE Procedure}

The SPE sample preparation procedure was conducted as follows. Aqueous solutions of arylamines were adjusted to $\mathrm{pH} 10$ with $0.1 \mathrm{M} \mathrm{K}_{2} \mathrm{CO}_{3}$. A disposable ODS reversed phase cartridge was activated by rinsing with $5 \mathrm{~mL}$ of methanol, which was subsequently displaced with $3 \mathrm{~mL}$ of water. $30-200$ milliliters of the aqueous sample were added and allowed to percolate slowly through the activated cartridge at a flow rate of approximately $10 \mathrm{~mL} / \mathrm{min}$ using a glass syringe. The cartridge was then washed with $5 \mathrm{~mL}$ of distilled water (discarded) and then eluted with $1 \mathrm{~mL}$ of methanol. The eluate was analyzed using HPLC.

\section{Calibration}

Standard solutions containing mixtures of 10 arylamines were prepared at five concentration levels in the range of $0.1-100 \mathrm{mg} / \mathrm{L}$. This solutions were analyzed by HPLC with UV detection at $254 \mathrm{~nm}$. The compounds were identified and quantified by comparing their retention values and integrated peak areas with those of known external standards.

\section{RESULTS AND DISCUSSION}

\section{Chromatographic Conditions}

The mobile phase used for the separation of the arylamines was Acetonitrile-water solutions. The linear gradient programme is given in Table 1. The chromatogram of a standard mixture of 10 arylamines under the optimized chromatographic conditions are shown in Figure 1.

Table 1 Linear Gradient of the Mobile Phase

\begin{tabular}{ccc}
\hline Time & Acetonitrile, \% & Water, $\%$ \\
\hline 0.0 & 30 & 70 \\
8.0 & 30 & 70 \\
16.0 & 90 & 10 \\
20.0 & 90 & 10 \\
\hline
\end{tabular}




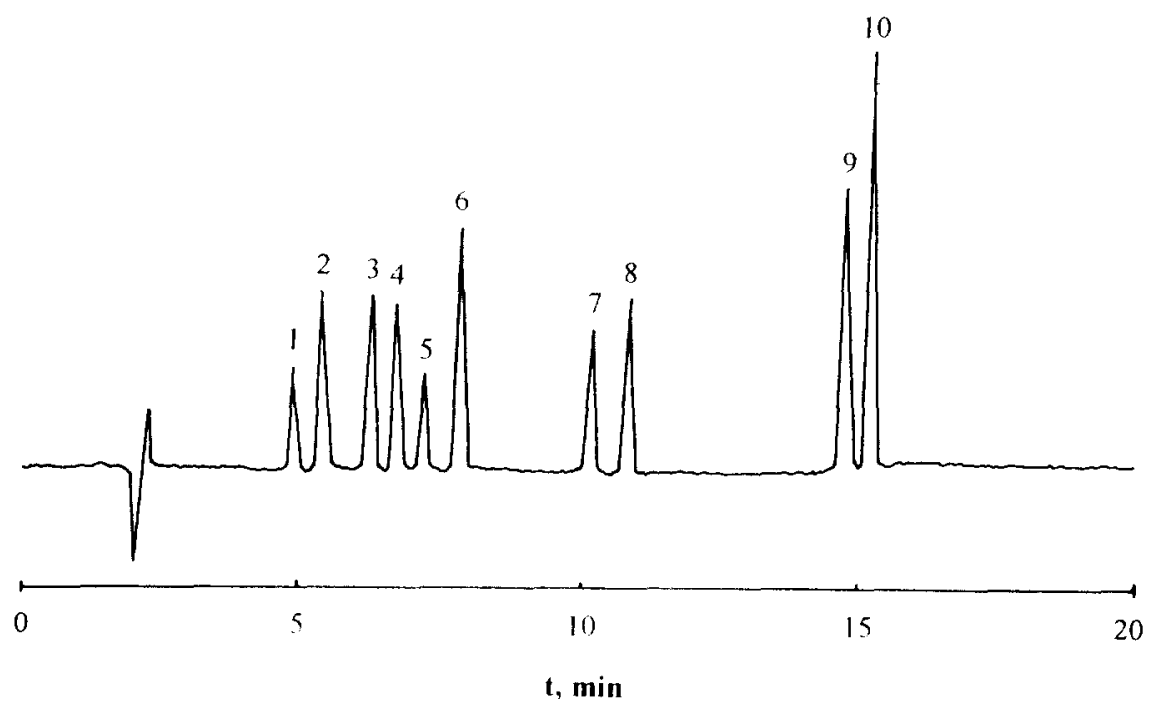

Figure 1: Chromatogram of a Standard Mixture of Ten Arylamines Conditions: Hypersil BDS (200×4.0 $\mathrm{mm}$ ) column; Mobile phase, gradient of acetonitrile/water, see Table 1; Flow rate, $0.8 \mathrm{~mL} / \mathrm{min}$; UV detection, $254 \mathrm{~nm}$; Injection volume, $10 \mu \mathrm{L}$. Peaks: 1 aniline; 2 benzidine; $3 . o$-anisidine; 4 quinoline; $5 o$ toluidine; 6. 4,4-methylenedianiline; 7. 3,3'-dimethoxybenzidine; 8. 3,3'-dimethylbenzidine; 9. 4aminobiphenyl; 10. N, N-dimethylaniline

\section{Optimization of SPE Conditions}

Methanol is often used as a solvent in SPE of polar compounds such as arylamines from water samples [8]. It was observed that best recoveries of arylamines were achieved with $1 \mathrm{~mL}$ of methanol. The optimal acidity of the extractive solutions prior to SPE was $\mathrm{pH} 10$. The selection of volume of water sample flow through the SPE cartridges indicated that the best recoveries were achieved with $200 \mathrm{~mL}$ of water samples for most of the arylamines studied. For the extraction of aniline, the recovery declined when the sample volume was more than $30 \mathrm{~mL}$, which is associated with the relatively high solubility of aniline in water.

\section{Retention Time, Linearity and Detection Limit}

It is shown from Figure I that the ten arylamines of interest are well separated from one another. With the preconcentration step considered, the enrichment factor for each arylamine is 200 (30 for aniline) Arylamines in the concentration range of $0.5-200 \mu \mathrm{g} / \mathrm{L}(5-200 \mu \mathrm{g} / \mathrm{L}$ for aniline $)$ in water samples could be determined with relative standard deviations of $0.8-16.3 \%$ (Correlation coefficient $r \geq 0.995$ ). The retention times and detection limits of the separated arylamines are listed in Table 2 . The detection limits are based on the signal-to-noise ratio of $3: 1$. A relatively high detection limit $(1.5 \mu \mathrm{g} / \mathrm{L})$ was obtained for aniline due to its high solubility in water. For the other arylamines, the detection limits are in the range of $0.1-0.3 \mu \mathrm{g} / \mathrm{L}$. 
Table 2 Retention Time and Detection Limit

\begin{tabular}{lcc}
\hline \multicolumn{1}{c}{ Compound } & $\begin{array}{c}\text { Retention time } \\
(\mathrm{min})\end{array}$ & $\begin{array}{c}\text { Detection limit* } \\
(\mu \mathrm{g} / \mathrm{L})\end{array}$ \\
\hline Aniline & 4.9 & 1.5 \\
Benzidine & 5.5 & 0.1 \\
o-Anisidine & 6.4 & 0.3 \\
Quinoline & 6.9 & 0.3 \\
o-Toluidine & 7.4 & 0.3 \\
4,4'-Methylenedianiline & 8.2 & 0.2 \\
3,3'-Dimethoxybenzidine & 10.6 & 0.3 \\
3,3'-Dimethylbenzidine & 11.3 & 0.2 \\
4-Aminobiphenyl & 14.9 & 0.1 \\
N,N-Dimethylaniline & 15.3 & 0.1 \\
\hline
\end{tabular}

* Signal-to-noise ratio $=3: 1$.

\section{Determination of Arylamine Compounds in Wastewater}

The proposed method was used to determine the arylamines in a wastewater sample, collected from the aqueous effluent (after secondary treatment) of a dye processing plant in Dalian city. The concentrations of arylamines in the sampled wastewater are listed in Table 3 . In the chromatogram of the wastewater sample no significant interferences were observed. The mean recoveries $(n=5)$ of the arylamines in the fortified wastewater sample were in the range of $84-107 \%$, which indicated that the proposed procedure is suitable for determining arylamines in wastewater sample.

\section{CONCLUSIONS}

This study established a HPLC method for separating and determining 10 arylamines in wastewater samples. These compounds were separated by an SPE procedure using methanol as solvent. The SPE technique had the advantage of being a rapid, convenient and inexpensive method for the extraction and preconcentration of such complicated samples as wastewater. The developed chromatographic method allowed sensitive, selective determination of arylamines in wastewater samples at or below $\mu \mathrm{g} / \mathrm{L}$ level. 
Table 3 The Concentrations of Arylamines in Wastewater

\begin{tabular}{lcc}
\hline \multicolumn{1}{c}{ Compound } & $\begin{array}{c}\text { Concentration } \\
(\mu \mathrm{g} / \mathrm{L})\end{array}$ & $\begin{array}{c}\text { Mean recovery** } \\
(\%)\end{array}$ \\
\hline Aniline & 62 & 84 \\
Benzidine & 23 & 92 \\
o-Anisidine & $\mathrm{ND}^{*}$ & 107 \\
Quinoline & $\mathrm{ND}$ & 96 \\
o-Toluidine & 47 & 91 \\
4,4'-Methylenedianiline & $\mathrm{ND}$ & 92 \\
3,3'-Dimethoxybenzidine & $\mathrm{ND}$ & 97 \\
3,3'-Dimethylbenzidine & ND & 89 \\
4-Aminobiphenyl & ND & 92 \\
N.N-Dimethylaniline & 126 & 102 \\
\hline
\end{tabular}

* Not detected.

** Based on the fortification of the sample with $20 \mu \mathrm{g} / \mathrm{L}$ of each arylamine Relative standard deviations were less than $8 \%(n=4)$.

\section{ACKNOWLEDGMENTS}

We are gratefully indebted to Dr. Hui-Qing Wang who, collected the water samples and provided part of the arylamine solutions and to Dr. Fa-Sheng Li for his helpful discussion and comments.

\section{REFERENCES}

1. T. S. Scott, ('arcinogenic and Toxic Hazards of Aromatic Amines, Elsevier Publishing, New York (1962).

2. R. M. Riggin, C. C. Howard, Anal. (hem, 51(2), 210-214 (1979)

3. D. N. Armentrout, S. S. Cutie, J. ('hromatogr. Sci, 18, 370-374 (1980).

4. M. S Varney, M. R. Preston, J. Chromatogr., 348, 265-274 (1985).

5. A. Di Corcia, R. Samperi, Anal. ('hem., 62, 1490-1494 (1990).

6. Dj. Djozan, M. A. Faraj-Zadeh, ('hromatographia, 41(9/10), 568-572 (1995).

7. P. Vitali, E. Venturini, C. Bonora, R. Calori, R. Raffaelli, J. ( hromatogr., 660, $219-222$ (1994)

8. I. Liska, A. Kuthan, \%. ('hromatogr., 509, 123-134 (1990).

9. J. S. Fritz, P. J. Dumont, L. W. Schmidt, J. (hromatogr. A, 691, 133-140 (1995). 